\title{
Bioprosthetic valve fracture: a practical guide
}

\author{
Keith B. Allen ${ }^{1}$, Adnan K. Chhatriwalla ${ }^{1}$, John T. Saxon ${ }^{1}$, Chetan P. Huded ${ }^{1}$, Janarthanan Sathananthan ${ }^{2}$, \\ Tom C. Nguyen ${ }^{3}$, Brian Whisenant ${ }^{4}$, John G. Webb ${ }^{2}$ \\ ${ }^{1}$ Saint Luke's Mid America Heart Institute and University of Missouri-Kansas, City School of Medicine, Kansas City, Missouri, USA; ${ }^{2}$ Centre for \\ Cardiovascular Innovation and Centre for Heart Valve Innovation, St. Paul's Hospital and University of British Columbia, Vancouver, Canada; \\ ${ }^{3}$ UCSF, San Francisco, CA, USA; ${ }^{4}$ Intermountain Medical Center, Salt Lake City, UT, USA \\ Correspondence to: Keith B. Allen, MD. 4320 Wornall Road, Medical Plaza II, Suite 50, Kansas City, MO 64111, USA. Email: kallen2340@aol.com.
}

\begin{abstract}
Valve-in-valve transcatheter aortic valve replacement (VIV TAVR) is currently indicated for the treatment of failed surgical tissue valves in patients determined to be at high surgical risk for re-operative surgical valve replacement. VIV TAVR, however, often results in suboptimal expansion of the transcatheter heart valve (THV) and can result in patient-prosthesis mismatch (PPM), particularly in small surgical valves. Bioprosthetic valve fracture (BVF) and bioprosthetic valve remodeling (BVR) can facilitate VIV TAVR by optimally expanding the THV and reducing the residual transvalvular gradient by utilizing a high-pressure inflation with a non-compliant balloon to either fracture or stretch the surgical valve ring, respectively. This article, along with the supplemental video, will provide patient selection, procedural planning and technical insights for performing BVF and BVR.
\end{abstract}

Keywords: Surgical aortic valve replacement; transcatheter aortic valve; replacement; valve in valve; bioprosthetic valve fracture (BVF)

Submitted Jul 03, 2021. Accepted for publication Aug 18, 2021.

doi: 10.21037/acs-2021-tviv-25

View this article at: https://dx.doi.org/10.21037/acs-2021-tviv-25

\section{Introduction}

Heart teams are increasingly utilizing valve-in-valve transcatheter aortic valve replacement (VIV TAVR) to manage failed bioprosthetic surgical valves beyond the currently approved Food and Drug Administration indication for high-risk surgical patients only $(1,2)$. An Achilles' heel of VIV TAVR, particularly in small surgical valves, is the potential for patient-prosthesis mismatch (PPM). The risk of severe PPM, typically defined as a residual transvalvular gradient $>20 \mathrm{mmHg}$, is higher following VIV TAVR since the transcatheter heart valve (THV) is constrained by the surgical valve's true internal diameter (ID). In addition, suboptimal expansion of the THV following VIV TAVR may result in pin-wheeling of the THV leaflets and premature THV degeneration $(3,4)$.

While the short-term results of VIV TAVR have generally been good, the observed one-year mortality following VIV TAVR in the Valve-in-Valve International Data (VIVID) Registry was significantly higher in patients with small surgical valves (labeled surgical valve size $\leq 21 \mathrm{~mm}$ ) compared to intermediate ( $\geq 23$ and $\leq 25 \mathrm{~mm}$ ) or large $(\geq 27 \mathrm{~mm}$ ) surgical valves ( $25 \%$ vs. $18 \%$ vs. $7 \%, \mathrm{P}=0.001)$, respectively (5). PPM may be responsible for this finding, with pre-existing PPM of the failed prosthetic valve an independent predictor of increased mortality after VIV TAVR (6). Strategies to improve the hemodynamic results of VIV TAVR and reduce the risk of PPM include; implanting the THV high in the surgical valve, since lower implantation depths can result in suboptimal leaflet function and increase the risk of high residual transvalvular gradients (7-10). In addition, utilizing a supra-annular THV, particularly in small surgical valves, can also minimize the risk of PPM. Unfortunately, PPM has occurred with a wide variety of THV's and despite a perfect implantation technique (8,11-13).

Bioprosthetic valve fracture (BVF) $(14,15)$ and bioprosthetic valve remodeling (BVR) $(16,17)$ are techniques to facilitate VIV TAVR, in which a high-pressure balloon inflation is performed using a non-compliant balloon to either fracture the surgical valve ring or stretch the surgical 
valve ring or posts, permitting more optimal expansion of the THV and thereby, mitigating the risk of PPM following VIV TAVR. In vitro testing of bioprosthetic surgical valves has demonstrated that most, but not all, can be fractured or remodeled $(14,16)$. In addition, clinical experience has demonstrated the safety and efficacy of BVF/BVR in reducing PPM following VIV TAVR (13-15,18). Finally, the reduction in transvalvular gradients following BVF appears durable at one-year follow-up, although the impact this may have on mortality and THV durability remains unknown (19). Achieving successful and safe results with $\mathrm{BVF} / \mathrm{BVR}$ requires a clear understanding of the procedure, which this article and supplementary video will provide.

\section{Bioprosthetic fracture set up}

When first considering $\mathrm{BVF} / \mathrm{BVR}$, it is necessary to accurately identify the surgical valve and determine if it can be fractured or remodeled. Aortic surgical valves that can be fractured (along with their fracture threshold) include the Magna (22-24 Atm, Edwards Lifesciences), Magna Ease (18 Atm, Edwards Lifesciences), Perimount 2800 (20 Atm, Edwards Lifesciences), Mitroflow (12 Atm, Sorin Group), Mosaic (10 Atm, Medtronic), and Biocor Epic (8, Atm, Abbott) (14). Surgical valves that can be remodeled or stretched, but not fractured, include; Trifecta (Abbott), Carpentier-Edwards standard and supra-annular (Edwards Lifesciences), Inspiris (Edwards Lifesciences) and, Perimount 2700 (Edwards Lifesciences) (16,17). Finally, surgical valves that cannot be fractured or remodeled include the Hancock II (Medtronic) and Avalus (Medtronic) surgical valves (14).

BVF is performed using the following equipment: (I) a non-compliant balloon [True Dilatation or Atlas Gold (Bard, Murry Hill, NJ, USA) are the most commonly used]; (II) a high-pressure stopcock and tubing; (III) an indeflator and; (IV) a $60-\mathrm{mL}$ syringe with dilute contrast (20). After initiating rapid ventricular pacing, the non-compliant balloon is rapidly filled with dilute contrast from the $60 \mathrm{~mL}$ syringe and then pressurized using the indeflator until fracture occurs. Confirmation of fracture can be challenging; observing in real time the waist of the balloon release at the time of fracture or listening for an audible snap, are unreliable. The best confirmation that BVF has occurred includes observing an acute drop in the indeflator pressure near the fracture threshold for the surgical valve and feeling a vibration or shutter through the shaft of the non-compliant balloon.

The pressures required to achieve BVF go well beyond the rated burst pressure of current non-compliant balloons (21). Balloon failure occurs in approximately $10 \%$ of cases and can mimic successful BVF with a drop in indeflator pressure. If the indeflator pressure acutely drops prior to reaching the predicted BVF fracture threshold, balloon failure has likely occurred. Balloon failure can be from rupture, which is always apparent, or more commonly, from a pin hole leak, occurring where the catheter enters or exits the balloon. A balloon failure from a pin hole leak will cause an acute drop in the indeflator pressure and mimic valve fracture. Pin hole leaks, unlike ruptures, do not cause a vibration in the shaft of the non-compliant balloon thus, a drop in the indeflator pressure without a vibration felt in the balloon shaft indicates balloon failure, not fracture. In addition, if the indeflator pressure drops acutely below the anticipated fracture threshold for a given surgical valve and continued inflation does not result in a pressure increase, then balloon failure has also likely occurred from a pin hole leak. If balloon failure is confirmed by removing and examining the balloon under pressure, then a new balloon should be prepped and BVF performed again.

The variability in fracture thresholds among surgical valves is directly related to the material used in the surgical valve frame. Recently, it has come to our attention that the Mosaic valve has been manufactured with two different materials and behaves differently during BVF depending on the material used to manufacture the frame. In our initial bench testing, the frame of the Mosaic valve was made of Delrin (acetal homopolymer resin) which is easily fractured at approximately 10-12 ATM (14). Although reported by Medtronic, it is not widely known that Mosaic valves have also been manufactured using the high-performance thermoplastic polyetheretherketone (PEEK) (personal communication, Medtronic). PEEK, when used in large amounts, such as in Medtronic's Avalus valve, cannot be fractured. However, the smaller amount used in the Mosaic valve, while still not allowing it to be fractured, does allow it to be stretched. During BVF on a Mosaic valve, if the fracture threshold is reached ( 10-12 ATM) and nothing has happened then the frame may be made of PEEK; continue to increase the inflation device pressure and at about 18 ATM, the valve frame will begin to stretch. Continue the inflation to approximately 22 ATM at which point maximum expansion should be obtained.

\section{THV valve/balloon selection}

Initial in vitro testing demonstrated that BVF results 
in an increase of 3-4 $\mathrm{mm}$ in the internal diameter of surgical valves with labeled valve sizes of 19 and $21 \mathrm{~mm}$, respectively (14). Additional bench testing (unpublished) has demonstrated that expansion of $5 \mathrm{~mm}$ can be achieved in larger labeled valve sizes (23 and $25 \mathrm{~mm}$ ) and clinical experience $(13,16)$ suggests that a $6 \mathrm{~mm}$ increase in diameter can be obtained following BVF in larger $(\geq 27 \mathrm{~mm})$ surgical valves. The selection of balloon size when performing BVF is an important consideration in achieving optimal outcomes and is dependent on understanding how much expansion will occur following BVF. Although BVF can be performed with any balloon larger than the true ID of the surgical valve, this will result in higher fracture pressure, less optimal expansion of the TAVR prosthesis and may negatively impact valve hemodynamics. In a recent retrospective study, the use of a balloon that was at least $3 \mathrm{~mm}$ larger than the true ID of the surgical valve was an independent predictor of a lower final transvalvular gradient (18). Our prior advice to use a balloon $1 \mathrm{~mm}$ larger than the labeled valve size is no longer appropriate, rather, the balloon size should be determined by the THV used, the true ID of the surgical valve and its anticipated increase in diameter following fracture, the anatomy of the aortic root and LVOT, and the location of the coronary arteries.

Using non-compliant balloons to perform BVF that are larger than previously recommended may allow more optimal expansion of the THV to its nominal size and/or allow placement of a larger than previously recommended THV. Assuming the virtual valve to coronary (VTC) distance supports BVF, the following example illustrates the concept of using a larger balloon to fully expand the THV. For example, if the true ID of the failing surgical valve was $19 \mathrm{~mm}$ we would anticipate a $3-4 \mathrm{~mm}$ increase in diameter following BVF and would select a $23 \mathrm{~mm}$ THV for VIV TAVR. In the case of a $23 \mathrm{~mm}$ Sapien, we would then perform BVF with a $23 \mathrm{~mm}$ non-compliant balloon in order to fully expand the $23 \mathrm{~mm}$ THV. With a Sapien, the BVF balloon can be right sized to the THV (i.e., a $26 \mathrm{~mm}$ balloon can be used safely in a $26 \mathrm{~mm}$ Sapien). If in the prior example a $23 \mathrm{~mm}$ CoreValve was implanted both balloon size and balloon placement during BVF are critical to avoiding injury to the THV leaflets (14). With CoreValve, it is only safe to use a balloon that is at most $2 \mathrm{~mm}$ larger than the 'constrained area' of the THV and to position the shoulder of the balloon below the level of the constrained area on the CV (14). Thus, the largest balloon that should be used during BVF with a $23 \mathrm{~mm} \mathrm{CV}$ is $22 \mathrm{~mm}$, a $26 \mathrm{~mm} \mathrm{CV}$ is $24 \mathrm{~mm}$ and a $29 \mathrm{~mm} \mathrm{CV}$ is $25 \mathrm{~mm}$.
This example illustrates how 'upsizing' or placing a larger THV than recommended might be accomplished assuming coronary obstruction was not in play. If, for example, VIV is being performed on a larger surgical valve with a true ID of $21 \mathrm{~mm}$, you would anticipate an increase in diameter following BVF of 4-5 mm. Therefore a $26 \mathrm{~mm}$ Sapien THV could be implanted and fractured with a $26 \mathrm{~mm}$ non-compliant balloon or a $26 \mathrm{~mm}$ CoreValve could be implanted and fractured with an appropriately positioned $24 \mathrm{~mm}$ balloon.

Coronary obstruction is an elemental risk during VIV TAVR and this risk is augmented by the addition of BVF $(22,23)$. Mitigating this risk requires a detailed understanding of the anticipated expansion of the surgical valve following BVF and its effect on the VTC distance as well as the risk of sequestration at the sinotubular junction. Determining the 'virtual' THV to coronary distance with a virtual valve and anticipating the $\sim 4 \mathrm{~mm}$ increase in diameter of the surgical valve following BVF is an important procedural planning step for avoiding coronary obstruction. The use of coronary protection strategies to avoid coronary obstruction are sometimes needed during VIV TAVR even without the addition of BVF.

\section{Bioprosthetic fracture: before or after VIV TAVR}

The timing of BVF, either before or after TAVR, remains controversial (3). While performing BVF after VIV TAVR provides the most optimal expansion of the THV, it does expose the THV to the high-pressure balloon inflation, which could cause acute or subacute injury to the THV, impacting durability. On the other hand, performing BVF first may increase the risk of embolization of debris from the degenerated surgical valve and cause acute valvular insufficiency leading to hemodynamic instability. The timing of BVF and the potential effect of high-pressure balloon inflation on Sapien 3 THV leaflets was evaluated in an accelerated leaflet calcification animal model (24). No acute THV injuries and no difference in leaflet calcification were noted between the BVF before and after groups $(\mathrm{P}=0.8)$.

Our recommendation is to perform BVF after VIV TAVR, which we feel is safer and also, ensures optimal expansion of the THV. In the case of balloon expandable THV's, the compliant delivery balloon is not robust enough to fully expand the THV in the already fractured surgical valve (14). When BVF is performed after TAVR with a balloon expandable THV, the non-compliant balloon both 
fractures the surgical valve and fully expands the already implanted THV (14). In the case of a self-expanding $\mathrm{THV}$, in vitro bench testing suggested it was capable of full expansion in a previously fractured non degenerated surgical valve (14). In vivo however, the self-expanding THV may not have enough radial force to fully expand a degenerated and calcified surgical valve, even if already fractured (18). When using a self-expanding THV, if BVF is performed first a high-pressure balloon inflation may still be required to achieve optimal expansion of the THV although at a lower pressure than was required to fracture the surgical valve initially (18). In a multivariable analysis of 75 BVF cases, performing BVF after performing VIV TAVR was an independent predictor $(\mathrm{P}<0.0001)$ of lower final mean transvalvular gradient, presumably due to more optimal expansion of the THV (18).

One question that is often asked is whether BVF can be performed weeks or even months after the initial VIV TAVR if transvalvular gradients are noted to be increasing. Assuming that rising gradients are not felt to be related to leaflet thrombosis, anecdotal reports suggest that late BVF (up to one year) can be successfully performed for patients who experience deterioration in initially favorable valve hemodynamics following VIV TAVR $(20,25,26)$. While failure to optimally expand the THV at the initial VIV TAVR might be corrected with delayed BVF, the damage to the leaflets that may have occurred in the interim is unlikely to be reversed by delayed BVF, potentially leading to premature failure of the THV. This raises the question as to whether BVF should be performed in all VIV cases where the surgical valves can be fractured regardless of the final mean gradient, in order to optimally expand the THV; this is our current practice.

BVF was initially conceived as a solution to high residual gradients following VIV TAVR however, optimal expansion of the THV may be just as important. Suboptimal expansion of a THV following VIV TAVR may lead to leaflet dysfunction and 'pin wheeling', which may decrease THV durability (4). Pin-wheeling refers to the 'pleated' appearance of the free edges of the redundant leaflets caused by under expansion of the valve (27). Pinwheeling which is often seen in the leaflets of a constrained THV may result in premature degeneration of the bioprosthetic leaflets, even in the setting of normal residual transvalvular gradients $(26,28)$. Bench testing has demonstrated that BVF reduces pinwheeling through optimal expansion of the THV (3). We currently perform BVF on all valves that can be fractured where the THV is not optimally expanded even if residual gradients are not high in order to optimize leaflet function. This might provide a THV durability benefit in all patients who undergo VIV TAVR, regardless of the size of the surgical valve or risk of PPM. However, this hypothesis will require further study and longer follow-up.

\section{Safety of BVF}

Complications have been reported following BVF which have included annular rupture, acute damage to the THV, damage to the mitral valve, THV embolization, iatrogenic ventricular septal defect, atrioventricular (AV) block requiring permanent pacemaker, coronary artery obstruction and stroke (29). Annular rupture is a significant concern with BVF but has been rarely reported (18). The ability to fracture and expand a surgical valve without injury to the native annulus is likely related to the fact that most surgical valves are implanted supra-annular rather than intra-annular and, that calcium is debrided from the annulus and left ventricular outflow tract (LVOT) at the time of surgery allowing expansion of the surgical valve into the aortic sinuses. However, the anatomy of the aortic root and LVOT must be assessed before BVF is considered. We do not recommend BVF in valves implanted intra-annularly, such as rapid deployment valves, or in patients that have had a root replacement or formal root enlargement. In many instances, complications occurred early in the BVF experience and could have been avoided with more appropriate BVF technique as outlined previously. In a recent report from twenty-one experienced TAVR centers who performed BVF in seventy-five patients, there were no coronary occlusions, no annular ruptures and no new pacemakers with durability of results persistent at one-year $(18,19)$.

\section{Bioprosthetic fracture: future studies}

While the focus of BVF has been in the aortic space, BVF may have utility in VIV pulmonary, mitral and tricuspid cases as a means to potentially reduce the incidence of VIV induced PPM (17,30-34). Although bench studies and clinical experience with BVF in the aortic position is known, particularly in small surgical valves, this knowledge may not extrapolate to larger valves that are frequently used in the tricuspid and mitral position. While safety concerns and efficacy with BVF in VIV mitral and tricuspid cases requires further study, there are also practical concerns to doing BVF in these larger valves. 
Currently, the largest non-compliant balloon available for performing $\mathrm{BVF}$ has a diameter of $28 \mathrm{~mm}$. In the aortic position, the availability of balloon sizes is adequate, as even in the largest surgical aortic valves, the true ID is exceeded by the true ID of the non-compliant balloon. This, however, is not the case with mitral and tricuspid valves, where the true ID of the surgical valve is often larger than the largest non-compliant balloon. While the bench top use of a 'kissing balloon' technique to fracture larger surgical valves is feasible, it is not very clinically practical (14). In addition to not having large enough balloons, the manufacturing parameters for non-aortic surgical valves are often dissimilar to their matched aortic counterparts. For example, a $27 \mathrm{~mm}$ aortic Magna Ease has a true ID of $25 \mathrm{~mm}$ and can be fractured with a $28 \mathrm{~mm}$ noncompliant balloon. On the other hand, a $27 \mathrm{~mm}$ mitral Magna Ease has a true ID of $26 \mathrm{~mm}$ and while it has a similar-looking metal ring to the aortic valve, it is actually slightly thicker (personal communication, Edwards) and, cannot be fractured with current balloons. Industry support from THV and balloon manufactures is needed to better understand such limitations of BVF and to optimize the procedure (35).

\section{Conclusions}

BVF, as an adjunct to VIV TAVR, appears safe when performed correctly and results in an unconstrained THV with improved hemodynamics, particularly; in small surgical valves thus, reducing the risk of severe PPM. Where feasible, we perform BVF in all cases regardless of the presence or severity of the residual gradient following VIV TAVR to optimally expand the THV. However, the BVF's effect on THV durability and long-term clinical outcomes requires further investigation. The expansion of BVF into the VIV mitral and tricuspid space, where even small reductions in gradients may yield significant benefits, is the next frontier that is just beginning to be explored.

\section{Acknowledgments}

Funding: None.

\section{Footnote}

Conflicts of Interest: KBA: Edwards Lifesciences: Research Support, Proctor, Speakers Bureau; Medtronic: Research Support, Speakers Bureau; Abbott: Research Support,
Consulting; Boston Scientific: Consulting; AKC: Abbott Vascular: Speakers Bureau; Boston Scientific: Research support, consulting; Edwards Lifesciences: Proctor, Speakers Bureau; Medtronic Inc: Proctor, Speakers Bureau. JTS: Medtronic Inc: Proctor. CPH: none. JS: Edwards Lifesciences and Medtronic: Consulting; TCN: Edwards Lifesciences: Consulting. BW: Edwards Lifesciences: Consulting. JGW: Consulting for Edwards Lifesciences, Abbott, Boston Scientific.

Open Access Statement: This is an Open Access article distributed in accordance with the Creative Commons Attribution-NonCommercial-NoDerivs 4.0 International License (CC BY-NC-ND 4.0), which permits the noncommercial replication and distribution of the article with the strict proviso that no changes or edits are made and the original work is properly cited (including links to both the formal publication through the relevant DOI and the license). See: https://creativecommons.org/licenses/by-nc-nd/4.0/.

\section{References}

1. Webb JG, Mack MJ, White JM, et al. Transcatheter Aortic Valve Implantation Within Degenerated Aortic Surgical Bioprostheses: PARTNER 2 Valve-in-Valve Registry. J Am Coll Cardiol 2017;69:2253-62.

2. Dvir D, Webb J, Brecker S, et al. Transcatheter aortic valve replacement for degenerative bioprosthetic surgical valves: results from the global valve-in-valve registry. Circulation 2012;126:2335-44.

3. Sathananthan J, Fraser R, Hatoum H, et al. A bench test study of bioprosthetic valve fracture performed before versus after transcatheter valve-in-valve intervention. EuroIntervention 2020;15:1409-16.

4. Sathananthan J, Sellers S, Barlow AM, et al. Valve-in-Valve Transcatheter Aortic Valve Replacement and Bioprosthetic Valve Fracture Comparing Different Transcatheter Heart Valve Designs: An Ex Vivo Bench Study. JACC Cardiovasc Interv 2019;12:65-75.

5. Dvir D, Webb JG, Bleiziffer S, et al. Transcatheter aortic valve implantation in failed bioprosthetic surgical valves. JAMA 2014;312:162-70.

6. Pibarot P, Simonato M, Barbanti M, et al. Impact of Pre-Existing Prosthesis-Patient Mismatch on Survival Following Aortic Valve-in-Valve Procedures. JACC Cardiovasc Interv 2018;11:133-41.

7. Simonato M, Azadani AN, Webb J, et al. In vitro evaluation of implantation depth in valve-in-valve using 
different transcatheter heart valves. EuroIntervention 2016;12:909-17.

8. Simonato M, Webb J, Kornowski R, et al. Transcatheter Replacement of Failed Bioprosthetic Valves: Large Multicenter Assessment of the Effect of Implantation Depth on Hemodynamics After Aortic Valve-in-Valve. Circ Cardiovasc Interv 2016;9:e003651.

9. Sathananthan J, Fraser R, Kütting M, et al. Impact of implant depth on hydrodynamic function of the ALLEGRA bioprosthesis in valve-in-valve interventions. EuroIntervention 2020;15:e1335-42.

10. Sathananthan J, Sellers SL, Fraser R, et al. Impact of implant depth on hydrodynamic function with the ACURATE neo transcatheter heart valve following valve-in-valve transcatheter aortic valve replacement in Mitroflow bioprosthetic valves: an ex vivo bench study. EuroIntervention 2019;15:78-87.

11. Dvir D. Treatment of Small Surgical Valves: Clinical Considerations for Achieving Optimal Results in Valve-inValve Procedures. JACC Cardiovasc Interv 2015;8:2034-6.

12. Azadani AN, Jaussaud N, Matthews PB, et al. Valvein-valve implantation using a novel supravalvular transcatheter aortic valve: proof of concept. Ann Thorac Surg 2009;88:1864-9.

13. Chhatriwalla AK, Allen KB, Saxon JT, et al. Bioprosthetic Valve Fracture Improves the Hemodynamic Results of Valve-in-Valve Transcatheter Aortic Valve Replacement. Circ Cardiovasc Interv 2017;10:e005216.

14. Allen KB, Chhatriwalla AK, Cohen DJ, et al. Bioprosthetic Valve Fracture to Facilitate Transcatheter Valve-in-Valve Implantation. Ann Thorac Surg 2017;104:1501-8.

15. Nielsen-Kudsk JE, Christiansen EH, Terkelsen CJ, et al. Fracturing the Ring of Small Mitroflow Bioprostheses by High-Pressure Balloon Predilatation in Transcatheter Aortic Valve-in-Valve Implantation. Circ Cardiovasc Interv 2015;8:e002667.

16. Saxon JT, Allen KB, Cohen DJ, et al. Bioprosthetic Valve Remodeling (BVR) of Unbreakable Surgical Valves During Transcatheter Valve-in-Valve Replacement (VIV TAVR). Struct Heart 2020;4:99-104.

17. Chhatriwalla AK, Sorajja P. Expanding Indications for Bioprosthetic Valve Fracture and Bioprosthetic Valve Remodeling. Circ Cardiovasc Interv 2018;11:e007017.

18. Allen KB, Chhatriwalla AK, Saxon JT, et al. Bioprosthetic valve fracture: Technical insights from a multicenter study. J Thorac Cardiovasc Surg 2019;158:1317-28.e1.

19. Chhatriwalla A, Allen KB, Saxon JT, et al. 1-Year Outcomes following Bioprosthetic Valve Fracture to
Facilitate Valve-in-Valve Transcatheter Aortic Valve Replacement. Struct Heart 2021;5:312-8.

20. Saxon JT, Allen KB, Cohen DJ, et al. Bioprosthetic Valve Fracture During Valve-in-valve TAVR: Bench to Bedside. Interv Cardiol 2018;13:20-6.

21. Sathananthan J, Hensey M, Sellers S, et al. Performance of the TRUE dilatation balloon valvuloplasty catheter beyond rated burst pressure: A bench study. Catheter Cardiovasc Interv 2020;96:E187-95.

22. Dvir D, Leipsic J, Blanke P, et al. Coronary obstruction in transcatheter aortic valve-in-valve implantation: preprocedural evaluation, device selection, protection, and treatment. Circ Cardiovasc Interv 2015;8:e002079.

23. Ribeiro HB, Rodés-Cabau J, Blanke P, et al. Incidence, predictors, and clinical outcomes of coronary obstruction following transcatheter aortic valve replacement for degenerative bioprosthetic surgical valves: insights from the VIVID registry. Eur Heart J 2018;39:687-95.

24. Allen KB, Chhatriwalla AK, Yousef A, et al. Bioprosthetic Valve Fracture Performed Before Versus After VIV TAVR: Impact of High-pressure Balloon Inflation on Leaflet Calcification. ISMICS Virtual 2020. Available online: https://meetings.ismics.org/abstracts/2020/C9.cgi

25. Kaur NJ, Bojar R, Hannan JC. Bioprosthetic Valve Fracturing: Better Late Than Never. Struct Heart 2019;3:441.

26. Maleki H, Shahriari S, Labrosse M, et al. Effect of Aortic Annulus Size and Prosthesis Oversizing on the Hemodynamics and Leaflet Bending Stress of Transcatheter Valves: An In Vitro Study. Can J Cardiol 2015;31:1041-6.

27. Stanová V, Zenses AS, Thollon L, et al. Effects of hemodynamic conditions and valve sizing on leaflet bending stress in self-expanding transcatheter aortic valve: An in vitro study. Artif Organs 2020;44:E277-87.

28. Midha PA, Raghav V, Condado JF, et al. Valve Type, Size, and Deployment Location Affect Hemodynamics in an In Vitro Valve-in-Valve Model. JACC Cardiovasc Interv 2016;9:1618-28.

29. Saxon JT, Allen KB, Cohen DJ, et al. Complications of bioprosthetic valve fracture as an adjunct to valve-in-valve TAVR. Struct Heart 2019;3:92-9.

30. Brown SC, Cools B, Gewillig M. Cracking a tricuspid perimount bioprosthesis to optimize a second transcatheter sapien valve-in-valve placement. Catheter Cardiovasc Interv 2016;88:456-9.

31. Hensey M, Alenezi AR, Murdoch DJ, et al. Transcatheter Tricuspid Valve-in-Valve Replacement With Subsequent 
Bioprosthetic Valve Fracture to Optimize Hemodynamic Function. JACC Cardiovasc Interv 2018;11:2226-7.

32. Kamioka N, Corrigan F, Iturbe JM, et al. Mitral Bioprosthetic Valve Fracture: Bailout Procedure for Undersized Bioprosthesis During Mitral Valve-inValve Procedure With Paravalvular Leak Closure. JACC Cardiovasc Interv 2018;11:e21-2.

33. Kaneko T, Piccirillo B, Golwala H, et al. Balloon Fracture of a Surgical Mitral Bioprosthesis During Valve-in-Valve Transcatheter Mitral Valve Replacement: First-in-Human

Cite this article as: Allen KB, Chhatriwalla AK, Saxon JT, Huded CP, Sathananthan J, Nguyen TC, Whisenant B, Webb JG. Bioprosthetic valve fracture: a practical guide. Ann Cardiothorac Surg 2021;10(5):564-570. doi: 10.21037/acs-2021tviv-25
Report. Circ Cardiovasc Interv 2018;11:e006273.

34. Shahanavaz S, Asnes JD, Grohmann J, et al. Intentional Fracture of Bioprosthetic Valve Frames in Patients Undergoing Valve-in-Valve Transcatheter Pulmonary Valve Replacement. Circ Cardiovasc Interv 2018;11:e006453.

35. Allen KB, Chhatriwalla AK, Saxon MD, et al. Bioprosthetic Valve Fracture: A Practical Guide to Facilitate Valve-In-Valve TAVR. Available online: https:// doi.org/10.1053/j.optechstcvs.2020.12.007 